\title{
7 Empfehlungen
}

Angesichts der vorab beschriebenen Situation hat die Akademie der Wissenschaften in Hamburg das Thema „Antibiotika-Resistenz“ aufgegriffen und gemeinsam mit der Nationalen Akademie der Wissenschaften Leopoldina am 25. und 26. Februar 2011 einen Workshop mit dem Thema „Warum brauchen wir neue Antibiotika (und bekommen keine)?“ veranstaltet. Die Arbeitsgruppe „Infektionsforschung und Gesellschaft“ der Akademie der Wissenschaften in Hamburg und Experten der Nationalen Akademie der Wissenschaften Leopoldina führten im Anschluss daran die Ergebnisse des Workshops in der vorliegenden Stellungnahme zusammen. Die Akademien geben die folgenden Empfehlungen.

\section{Empfehlung 1: Stärkung der Grundlagenforschung}

Eine breit angelegte Grundlagenforschung zur Entstehung, Verbreitung und Verhinderung von Resistenzbildung sowie zur Entwicklung neuer Antibiotika ist unabdingbar. Die in dieser Stellungnahme diskutierten Forschungsfelder sollten prioritär bearbeitet werden. Dabei kommt den Methoden der klassischen Mikrobiologie eine große Bedeutung zu.

Besonders wichtige Punkte sind:

- Identifizierung neuer Targets mittels funktioneller Genomforschung und metagenomischer Ansätze

- Entwicklung neuer und effektiverer Screeningmethoden und Aufbau leistungsfähiger Substanzbibliotheken

- Isolierung und Züchtung von Mikroben, u.a. aus Umwelthabitaten als Quelle neuer Wirkstoffe

- Analyse zur Bedeutung des Wirtsmikrobioms (Metagenom) bei der Entstehung und Weitergabe von Resistenzen

- Aufklärung der klinischen und molekularen Mechanismen der Resistenz in vivo

\section{Empfehlung 2: Verbesserung der strukturellen Voraussetzungen für Innovationen}

Bevorzugt sollten Antibiotika entwickelt werden, die neue Zielstrukturen angreifen oder bislang nicht beteiligte Stoffwechselwege hemmen. Dabei kommt der Entwicklung einer stabilen Produkt-„Pipeline“ eine besondere Bedeutung zu. Eine dafür notwendige Voraussetzung ist der Erhalt und Ausbau der Infrastruktur zur Erforschung und Entwicklung neuer Antibiotika. Ferner ist es wichtig, Kooperationen zwischen Industrie und akademischer Forschung zu erleichtern und zu stärken, um Ressourcen 
der Grundlagenforschung effizienter mit den vielfältigen Anforderungen der pharmazeutischen Produktentwicklung zu verknüpfen.

Unabdingbar ist auch die weitere internationale Koordination von Maßnahmen zwischen Regierungen und der Industrie. Öffentliche Anreizmodelle sollten etabliert werden, um eine Rückkehr der Industrie zur Erforschung und Entwicklung von Antibiotika zu fördern. Um die Industrie dazu zu veranlassen, genügend Reserve-Antibiotika vorzuhalten, sollten sogenannte „Rückhalteprämien“ eingeführt werden. Die finanziellen Risiken für die aufwändigen und teuren klinischen Phase-IIIStudien sollten gemeinsam von Industrie und öffentlicher Hand getragen werden.

Trotz der bereits existierenden Netzwerke ist mit Blick auf die Entwicklung neuer Antibiotika eine stärkere Förderung notwendig, welche es ermöglicht, Forschungsstrukturen über nationale Grenzen hinweg längerfristig zu etablieren. Insbesondere sollten die Projekte der letzten Jahre evaluiert und gute Ansätze weiter verfolgt werden.

\section{Empfehlung 3: Erleichterungen für die klinische Forschung}

Klinische Studien zur Dauer effektiver Antibiotika-Therapien, zum Nutzen unterschiedlicher Therapieregime und zum Einfluss auf die Resistenzentstehung sollten verstärkt durchgeführt und finanziell unterstützt werden. Eine zentrale Rolle bei der Überführung neuer Wirkstoffe in die klinische Anwendung spielen translationale Forschungsansätze, die ebenfalls stärker gefördert werden sollen.

Studien, die von unabhängigen Wissenschaftlern an universitären oder außeruniversitären Einrichtungen initiiert werden (Investigatorinitiated trials - IIT), sollten unkomplizierter und schneller geprüft und gegebenenfalls genehmigt und durch öffentliche Förderprogramme unterstützt werden.

Zentren für klinische Studien sollten die Ausbildung von Fachpersonal sicherstellen, die notwendige Infrastruktur und auch Fördermittel für erste klinische Studien bereitstellen. Die Mittelvergabe an solche Zentren klinischer Forschung sollte evaluiert werden, in Abhängigkeit von der Qualität und Innovationskraft der an diesen Zentren organisierten klinischen Forschung. Modelle der Private Public Partnership sollten weiterentwickelt werden, bei denen die Kosten klinischer Studien aus öffentlichen Mitteln gedeckt, aber bei kommerzieller Nutzung anteilsmäßig wieder zurückerstattet werden und die öffentlichen Fördereinrichtungen an den Erlösen angemessen beteiligt wird. 


\section{Empfehlung 4: Weiterentwicklung der regulatorischen Rahmenbedingungen}

Der Überlegenheitsnachweis bei neuen Antibiotika gegenüber zurzeit verfügbaren Substanzen ist aufgrund der beschriebenen Resistenzentwicklungen ein $\mathrm{zu}$ hohes Therapieziel. Stattdessen sollten mehrere Substanzen mit ähnlicher Wirksamkeit zur Verfügung stehen. Es sollte berücksichtigt werden, dass die zukünftige Resistenzentwicklung nicht kalkulierbar ist und dass individuell seltenere Risiken auftauchen könnten (z. B. Allergien, Medikamenteninteraktionen). Als Therapieziel für die Genehmigung insbesondere neuer Therapieprinzipien und neuer Substanzklassen sollte zukünftig ein Wirksamkeitsnachweis ausreichen.

Diese Problematik der Entwicklung von Antibiotika-Resistenzen sollte in den regulatorischen Anforderungen berücksichtigt werden. Die Zulassung neuer Antibiotika, zunächst nur im Rahmen von Kombinationsregimen, sollte ermöglicht werden, da dies dazu beitragen könnte, die Entwicklung von Resistenzen zu vermeiden oder zumindest zu verzögern. Regulatorische Vorschriften für die Entwicklung und Zulassung neuer Antibiotika sollten klar formuliert werden. Sehr zu begrüßen wären auch vereinfachte regulatorische Standards sowie ein beschleunigtes Zulassungsverfahren, insbesondere von Neuentwicklungen gegen besonders kritische Erreger.

\section{Empfehlung 5: Einschränkung des Einsatzes von Antibiotika in der Tiermedizin und im Pflanzenschutz}

Antibiotika sollten möglichst nur nach klinischer Diagnose und basierend auf den Ergebnissen von Resistenztests zielgerichtet eingesetzt werden. Dabei muss sichergestellt sein, dass der zu bekämpfende Erreger bakterieller Natur ist. Die Anwendung von Antibiotika sollte nur nach tierärztlicher Verordnung erfolgen. Tierpathogene Bakterien und Zoonose-Erreger sollten kontinuierlich überwacht werden. Die fortlaufende Erhebung von Resistenzdaten, wie sie in Deutschland bereits gängige Praxis ist, ist dabei auszuweiten. Die von der Bundesregierung im September 2012 vorgeschlagenen Maßnahmen zur Reduzierung des Antibiotika-Einsatzes in der Tierhaltung sind deshalb zu begrüßen. Insbesondere die Erfassung von Daten zur Therapiehäufigkeit in einer zentralen Datenbank gibt den Behörden ein Instrument an die Hand, welches es erstmalig ermöglicht, den Einsatzes unterschiedlicher Antibiotikagruppen zur Bekämpfung von Infektionen bei den unterschiedlichen Tierarten deutschlandweit zu erfassen.

Beschäftigte in der Landwirtschaft und in der Lebensmittelindustrie sollten im Rahmen von Fortbildungsmaßnahmen darüber aufgeklärt 
werden, wie Antibiotika-Resistenzen entstehen und welche Maßnahmen ihre Entstehung verringern. Intensiver erforscht werden sollte auch, welche Auswirkungen der Einsatz von Antibiotika in der Tierhaltung und im Pflanzenschutz auf die Entstehung und Ausbreitung Antibiotika-resistenter Bakterien hat und wie sich die Übertragung pathogener Bakterien von Nutztieren auf den Menschen auswirkt.

\section{Empfehlung 6: Konsequente Durchführung einer Surveillance, Antibiotika-Verbrauchserfassung und -reduktion, Förderung der Aus- und Weiterbildung}

Regelmäßig sollte eine Surveillance von Resistenzraten wichtiger Erreger auf allen Ebenen erfolgen: lokal bis weltweit und bereichsübergreifend in Klinik, Ambulanz und Tierzucht. Die Erhebungsdaten sollten jährlich veröffentlicht werden. Eine solche regelmäßige Erfassung bedarf einerseits der Kooperation der beteiligten Akteure auf allen Ebenen. Andererseits sollten standardisierte einheitliche Testsysteme und Grenzwerte für diagnostische Labors definiert und eingeführt werden. Neben pathogenen Erregern sollten auch kommensale Bakterien fortlaufend beobachtet werden. Behandlungsempfehlungen für den klinischen und ambulanten Bereich sollten durch die dafür zuständigen Gremien, insbesondere die Kommission für Antiinfektiva, Resistenz und Therapie (ART) beim Robert Koch-Institut, auf Basis der erhobenen Resistenzinformationen weiter erstellt und breit zugänglich gemacht werden.

Die Akademien begrüßen den mit der Deutschen Antibiotika-Resistenzstrategie (DART) eingeschlagenen Weg. Mit der Änderung des Infektionsschutzgesetzes im Sommer 2011 wurden notwendige Maßnahmen ergriffen, um Antibiotika rationaler einzusetzen und Infektionserkrankungen zu vermeiden. Diese Maßnahmen sollten konsequent fortgeführt werden und zur Reduktion nosokomialer Infektionen sowie zur Prävention von Infektionen beitragen. Sie sollten deshalb konsequent in ihrer Entwicklung und Umsetzung gefördert werden. Auswirkungen von Maßnahmen sollten verstärkt durch Monitoring-Aktivitäten dokumentiert und überprüft werden. Epidemiologische Studien und Untersuchungen zur Übertragung von Resistenzgenen sollten die Monitoring-Aktivitäten begleiten.

Insbesondere sollte der Verbrauch von Antibiotika im klinischen und ambulanten Bereich umfassend erfasst und analysiert werden. Prophylaktische Antibiotikagaben sollten reduziert, initial-adäquate Antibiotika-Therapien dagegen häufiger eingesetzt werden. Die Koordination und Veröffentlichung von Daten zum Verbrauch von Antibiotika und zu Resistenzen auf nationaler und EU-Ebene durch das Robert Koch-Institut 
bzw. durch das ECDC sollten weitergeführt und ausgebaut werden. Die Repräsentativität der Datengrundlage sollte verbessert werden.

Voraussetzung für einen besseren Umgang mit Antibiotika und für die Vermeidung bzw. Verzögerung von Resistenzen ist eine Sensibilisierung aller Beteiligten im Gesundheitswesen für das Thema AntibiotikaResistenzen. Daher sollten regelmäßige Fortbildungen eingeführt und spezifische Teams an Kliniken etabliert werden. Zudem sollte es Schulungen von Personal im Gesundheitswesen geben, die rationale AntibiotikaTherapien, ein besseres Verständnis von Resistenz-Mechanismen und die jeweils aktuelle Resistenz-Situation zum Thema haben.

\section{Empfehlung 7: Stärkung der sozio-ökonomischen Forschung}

Die sozio-ökonomischen, rechtlichen und ethischen Rahmenbedingungen für die Entwicklung neuer Antibiotika sollten stärker erforscht, Hemmnisse identifiziert und Lösungswege aufgezeigt werden. Maßnahmen sollten verstärkt vorausschauend und rückblickend evaluiert werden. Ferner kann sozio-ökonomische Forschung dazu beitragen, Maßnahmen zur schnelleren und effizienten Entwicklung und Anwendung von Antibiotika zu entwickeln. Weiterhin sollten Schwerpunkte auf das Design adäquater Anreizmechanismen und die Untersuchung ihrer Wirkungen gelegt werden, sowie auf ein besseres Verständnis des Entscheidungsverhaltens von Anwendern und Produzenten. Eine große Bedeutung für die schnelle Umsetzung wissenschaftlicher Erkenntnisse in die klinische Praxis kommt den klinischen Studien und der Versorgungsforschung zu.

\section{Empfehlung 8: Einrichtung eines Runden Tisches zu Antibiotika- Resistenzen und neuen Antibiotika}

Die Akademien empfehlen, einen Runden Tisch zu Antibiotika-Resistenzen und neuen Antibiotika unter dem Dach der Akademien der Wissenschaften unter Beteiligung des Deutschen Zentrums für Infektionsforschung (DZIF) zu etablieren. Als unabhängige Institutionen bieten die Akademien der Wissenschaften einen Rahmen dafür, gemeinsam mit den relevanten Akteuren aus Wissenschaft, Politik, Behörden und Industrie rechtzeitig auf Probleme hinzuweisen und Lösungen aufzuzeigen. Aufgabe des Runden Tisches könnte es sein, Themenfelder zu identifizieren, Handlungsbedarfe aufzuzeigen und die Forschungsagenda an aktuelle Entwicklungen anzupassen. 
